\title{
A technical note on the geometric effects of volume to surface mapping of fMRI data
}

\author{
Keith George Ciantar ${ }^{\mathrm{a}}$, Ting $\mathrm{Xu}{ }^{* \mathrm{~b}}$, Claude J. Bajada*a,c \\ ${ }^{a}$ Department of Physiology and Biochemistry, Faculty of Medicine and Surgery, The University of Malta \\ ${ }^{b}$ Child Mind Institute, New York, NY, USA \\ ${ }^{c}$ Institute of Neuroscience and Medicine (INM-1), Research Centre Juelich, 52425, Juelich, Germany
}

\begin{abstract}
In this technical report we identify and report a problem with the process of volume to surface mapping that has potentially impacted studies that focus upon local neighborhood connectivity. We show that neighborhood correlations vary spatially with anatomical patterns (gyral structure) even when the underlying volumetric data is uncorrelated noise. We explore the effects of this anomaly across varying data resolutions and surface mesh densities. We finally propose an approach to mitigate these unwanted effects.
\end{abstract}

Keywords: Functional MRI, volume to surface mapping, cortical organization

\section{Introduction}

Surface based functional MRI has become a popular approach for performing fMRI analysis. An increasing number of large projects provide surface-based data (e.g. Human Connectome Project [HCP] (Van Essen et al. 2012, 2013), Adolescent Brain Cognitive Development [ABCD] study (Bjork et al., 2017)) as well as 5 software packages (e.g. FreeSurfer or Connectome Workbench) to facilitate the surface analysis. Recent fMRI packages have also implemented surface-based analysis in the preprocessing pipelines (e.g. the HCP Pipelines or fmriPrep) (Esteban et al. 2019, Glasser et al., 2013). There are good theoretical (Brodoehl et al., 2020: Glasser et al., 2016) and practical advantages (Coalson et al., 2018) to performing fMRI analysis on a cortical surface mesh. The primary advantages are that smoothing on a two dimensional surface respects the geometry of the brain and reduces signal contamination from geodesically distant areas of cortex (yet close in euclidean distance - such as opposing banks of neighboring sulci) and crosss-subject alignment in $2 \mathrm{D}$ on the surface is fundamentally an easier problem than in $3 \mathrm{D}$ in the volume. As part of the surface based preprocessing pipeline, the user must first project their volumetric (voxel) data to surface vertices. The process itself, however, has the potential to introduce artefacts that may affect some downstream surface-based analyses.

In this technical note we identify and report a problem with the process of volume to surface mapping in the context of analysing local neighborhood connectivity. The effect has potentially impacted some studies that have not taken it into consideration.

\section{The Problem}

An anomaly was noted when analysing regional boundaries (or edges) in functional signatures across the cortex (Bajada et al. 2020). Specifically, when aiming to characterize the regional boundaries by calculating the local functional changes between neighborhood vertices along the surface, we observed a clear anatomical

\footnotetext{
* Corresponding author

Email addresses: xutingxt@gmail.com (Ting $\mathrm{Xu}^{*}$ ), claude.bajada@um.edu.mt (Claude J. Bajada*)
} 
pattern that follows the gyral boundaries of the brain, even when using stochastic data as an input (Figure 1). This suggests that the local connectivity analysis might be affected, at least partially, by the surface 25 geometry.

A related phenomenon was first noted by (Glasser et al., 2013) where they noted that increasing the resolution of functional MRI data decreases "geometric effects" on surface data. Their primary concern was, however, the effects on correlation between relatively distant (geodesically) cortical areas.

In order to explore the phenomenon as it relates to neighborhood correlations more rigorously, uncorre30 lated noise (drawn from a standard normal distribution) was used to create synthetic fMRI timeseries at the voxel level. These were then projected to the surface using various volume-to-surface mapping approaches.

To simplify interpretations for this report, we first defined a local region that includes a given vertex and its neighborhood of vertices, one which shares an edge on the cortical mesh. Next, we calculated the pairwise Pearson correlations between any two vertices within the local region. Finally, we performed the

Fisher-z transform and used the mean of z-scores as the local connectivity index for the given vertex.

Similar patterns, following the gyrification patterns, that were observed with the real data were found. This prompted a more systematic exploration of the issue with the aim to understand and mitigate the problem.

\section{Methodology}

The T1-weighted data of a single subject from the minimally preprocessed Human Connectome Project Young Adult data-set were used for this study (Glasser et al., 2013). These data were selected in order to ensure high quality results and ensure state-of-the-art preprocessing up to the point of encountering the discussed issue. Noise timeseries were generated at three isotropic resolutions $(0.7 \mathrm{~mm}, 1.4 \mathrm{~mm}$ and $2 \mathrm{~mm})$ in volumetric space and pushed to the surface using five different volume to surface algorithms available within Connectome Workbench. The first two used simple trilinear and cubic interpolation at the level of the midthickness surface. The second two used the advanced ribbon constrained algorithm which uses constructed polyhedra constrained by the pial and white matter surface and uses the volume to weight the underlying voxel contribution (including a variant in which non-overlapping polyhedra are used, designated "thin columns" (TC)). The final approach termed the "enclosing" approach simply uses the voxel beneath the vertex of the midthickness surface. In order to isolate the problem as the interpolation between the voxel data and the surface, random noise was also drawn independently for each vertex in the reconstructed surface, we refer to this in figures as "pure noise".

To ensure a rigorous evaluation of the effect all of the different approaches of volume to surface mapping we carried out the following:

1. Volume to surface mapping of noise timeseries using the five approaches onto the native high-resolution surface (in the MNI standard space as per HCP data). Then down-sampling the maps to the surface resolution of interest (32k and 10k). We term this approach the "native mesh approach";

2. Volume to surface mapping using the five approaches directly onto the resolution of interest (native, $32 \mathrm{k}$ and $10 \mathrm{k}$ ) surfaces. We term this approach the "direct low resolution approach";

3. Volume to surface mapping using the three more basic approaches (trilinear, cubic and enclosing), onto midthickness surface meshes that approximate the resolutions of interest but that have undergone modification to reduced the variance of the inter-vertex distances (Attene, 2010);

4. Scatter plots showing the relationship between mean distance of neighborhood vertices and local correlations were plotted.

5. Histograms of the local correlations were also plotted for all of the different approaches.

For the sake of brevity, only the most pertinent results are discussed in the following section, however, all results and code are made available for further exploration and scrutiny (https://osf.io/dxr6f/). 


\section{Results}

A clear relationship between volume voxel size and surface resolution was found. As surface resolution (vertex density) is increased, or underlying voxel data resolution decreased, more surface vertices sample the same voxels from the volume. This results in an artefactual local correlation despite the original, volumetric data being uncorrelated noise. We note that this issue should not be unique to brain data or surface-based processing, upsampling any data to a higher sampling density should present a similar issue. Figure 2 shows, in the direct low resolution approach, the neighborhood correlations are approximately zero until the mean neighborhood distance decreases below a certain range (approximately equal to the resolution of the volume data). This pattern is replicated if the native mesh approach is used (see supplementary data and figures).

\section{Mitigation}

From the results of our investigations two points emerge (in the context of analyzing local correlations):

1. underlying voxel data should be of an equal or higher resolution relative to the surface mesh density. The important point is that the underlying data should not be of lower resolution than the surface mesh density;

2. any variance in intra-vertex distance on the mesh will create systematic "problematic areas." As a result, the uniformisation of surface meshes may mitigate the problem.

We ran the same pipeline on uniform meshes. As can be seen in Figures 4 and 5 , the effect on projecting

$2 \mathrm{~mm}$ data (typical high quality fMRI resolution) onto a 10k uniform surface greatly improves the local connectivity estimation of the data. Figure 5 shows that the noise surface to volume mapping does not show any obvious geometric patterns in local correlations. Further, additional analysis using real fMRI data from resting state and motor conditions show high local correlations in areas that are plausible for the specific task. It is important to note, however, that this approach comes at a cost of 1) decreasing spatial resolution

90 2) affecting SNR and 3) requires additional steps in order to regain vertex correspondence to a standard template.

\section{Conclusion}

In this technical report, we note that:

1. the relative difference between the cortical surface vertex density and underlying voxel resolution is important to consider;

2. not considering the variability of oversampling during volume to surface mapping to a relatively dense mesh (in relation to the data resolution) has the potential to affect studies that are interested in local neighborhood connectivity;

3. the artefactual geometric patterns that follow the cortical gyrification pattern can be mitigated (but not necessarily solved) by a) mapping data to a lower resolution mesh and b) ensuring that the mesh has a relatively low intra-vertex distance variance.

Overall, the present study highlights that geometric effects occur in surface based analysis investigating local neighborhood connectivity. As such any researcher that is interested in these investigations should take such effects seriously.

Data are available in a Jupyter notebook that can be used to recreate all of the analysis shown in this report. Additional figures that were not displayed due to limitations of the article format are also available in the supplementary material 
bioRxiv preprint doi: https://doi.org/10.1101/2020.11.09.373860; this version posted November 9, 2020. The copyright holder for this preprint (which was not certified by peer review) is the author/funder, who has granted bioRxiv a license to display the preprint in perpetuity. It is made available under aCC-BY-NC-ND 4.0 International license.

\section{Acknowledgements}

We acknowledge funding from NIMH (R24MH114806, R24MH117428-01). Data were provided by the Human Connectome Project, WU-Minn Consortium (Principal Investigators: David Van Essen and Kamil Ugurbil; 1U54MH091657) funded by the 16 NIH Institutes and Centers that support the NIH Blueprint for Neuroscience Research; and by the McDonnell Center for Systems Neuroscience at Washington University. The authors would like to acknowledge Lucas Q. Costa Campos for useful discussion on multiple versions of this manuscript. We would also like to thank Alexander Opitz and Michael Milham for useful suggestions in the initial investigation.

\section{Figures}

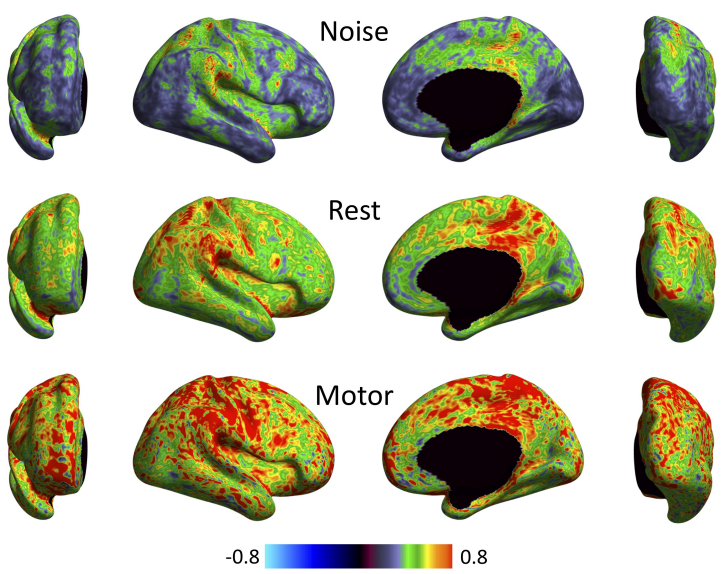

Figure 1: A delineation of the observed anomaly. The maps show, surface based, vertex-wise neighborhood correlation maps on three separate data sets. The top figure shows the map computed on data where the underlying volume was comprised of noise timeseries which was subsequently projected to a surface. The middle map was computed on resting state surface projected data and the bottom image was computed on a motor task dataset. Note that the middle image was rotated to be comparable with figure 5 
bioRxiv preprint doi: https://doi.org/10.1101/2020.11.09.373860; this version posted November 9, 2020. The copyright holder for this preprint (which was not certified by peer review) is the author/funder, who has granted bioRxiv a license to display the preprint in perpetuity. It is made available under aCC-BY-NC-ND 4.0 International license.
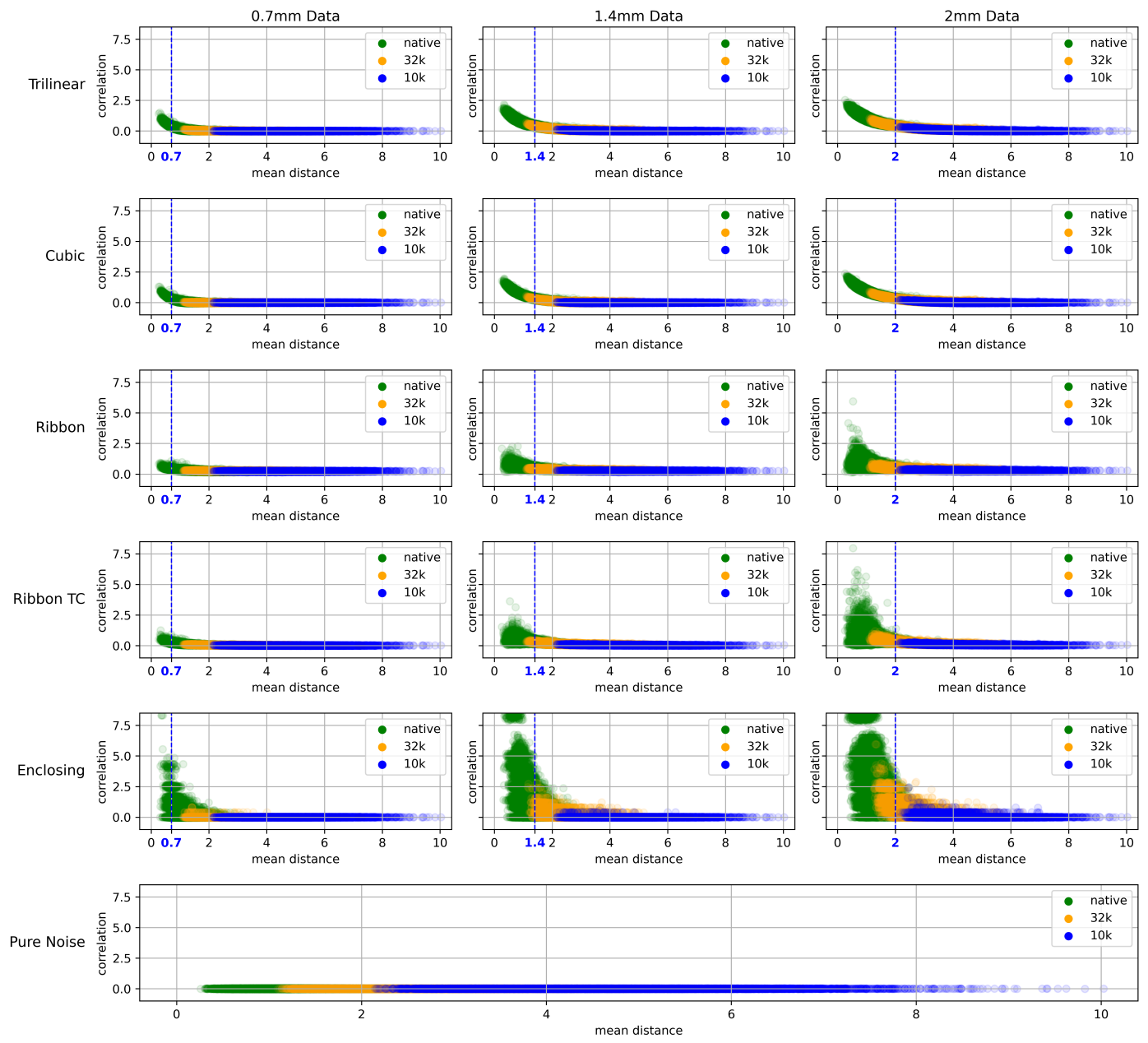

Figure 2: Matrix of various direct low resolution mapping approaches with various underlying voxel resolution. The bottom graph shows what is expected if local correlations are computed on "pure" noise timeseries that were generated directly on a surface mesh (with no volume to surface mapping artefacts. Colours indicate different mesh density, blue $=10 \mathrm{k}$, yellow $=32 \mathrm{k}$ and green $=$ native. The colours are consistently applied across all figures in this article and in the supplementary material. Medial wall voxels excluded from plots. The vertical blue line represents the data resolution in a single dimension. Note that more detailed, "individual mesh" level charts are available in the supplementary material. 
bioRxiv preprint doi: https://doi.org/10.1101/2020.11.09.373860; this version posted November 9, 2020. The copyright holder for this preprint (which was not certified by peer review) is the author/funder, who has granted bioRxiv a license to display the preprint in perpetuity. It is made available under aCC-BY-NC-ND 4.0 International license.
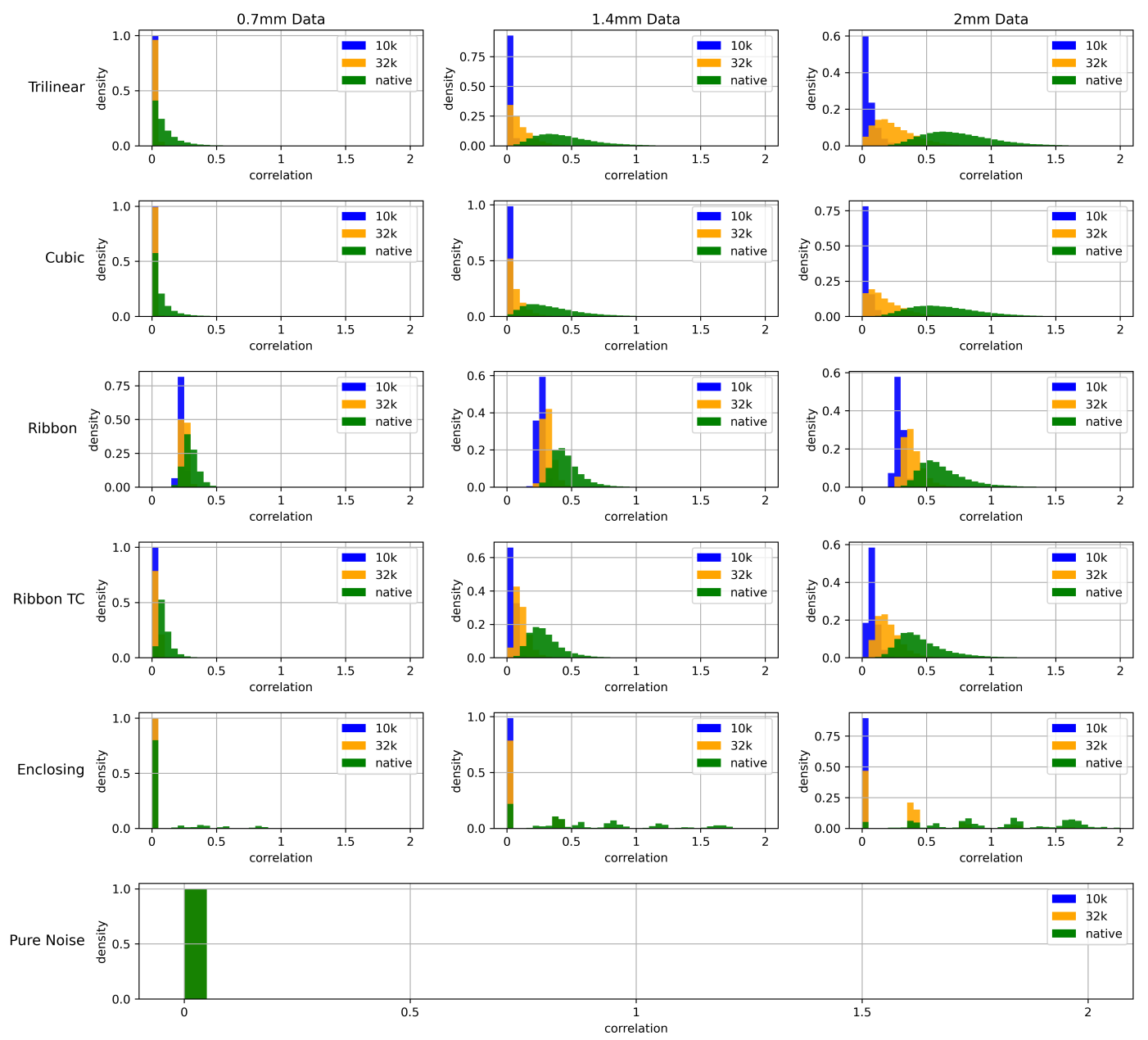

Figure 3: Matrix showing histograms of the various direct low resolution mapping approaches with various underlying voxel resolution. Colours indicate different mesh density, blue $=10 \mathrm{k}$, yellow $=32 \mathrm{k}$ and green $=$ native (midline voxels excluded from plots) 
bioRxiv preprint doi: https://doi.org/10.1101/2020.11.09.373860; this version posted November 9, 2020. The copyright holder for this preprint (which was not certified by peer review) is the author/funder, who has granted bioRxiv a license to display the preprint in perpetuity. It is made available under aCC-BY-NC-ND 4.0 International license.
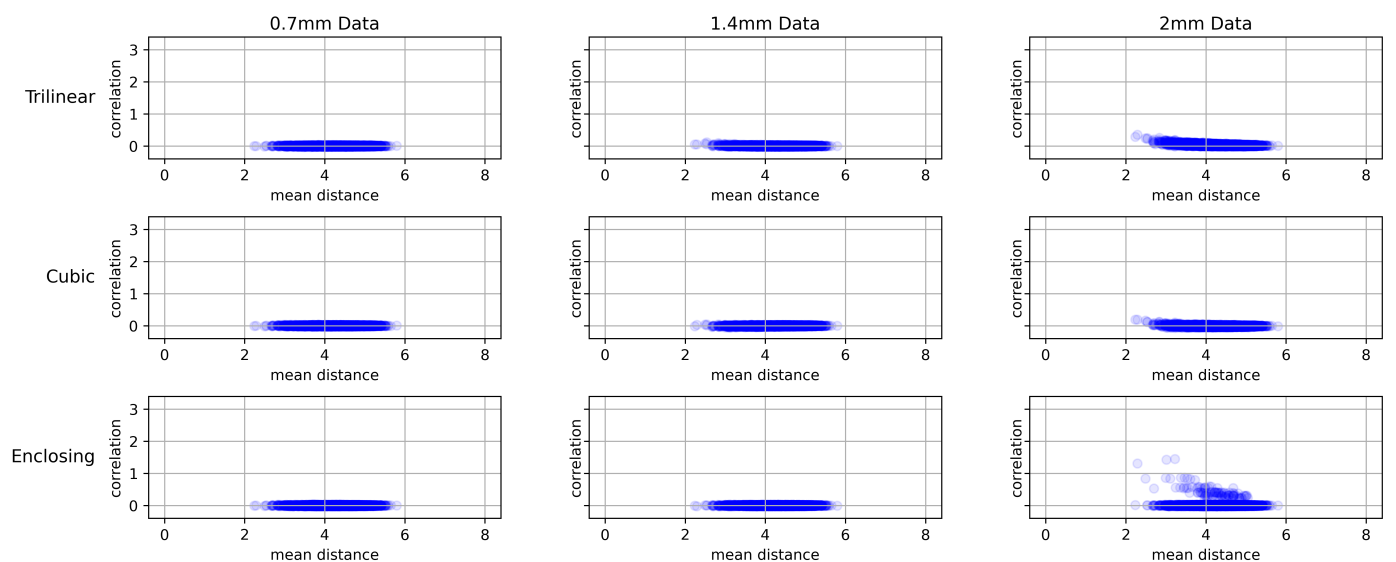

Figure 4: The impact of uniformisation of the mesh. Across all three volume to surface approaches and across all volumetric resolutions at a surface resolution of $10 \mathrm{k}$ vertices, the neighborhood correlations remain around zero (except for some neighborhoods in the enclosing approach).

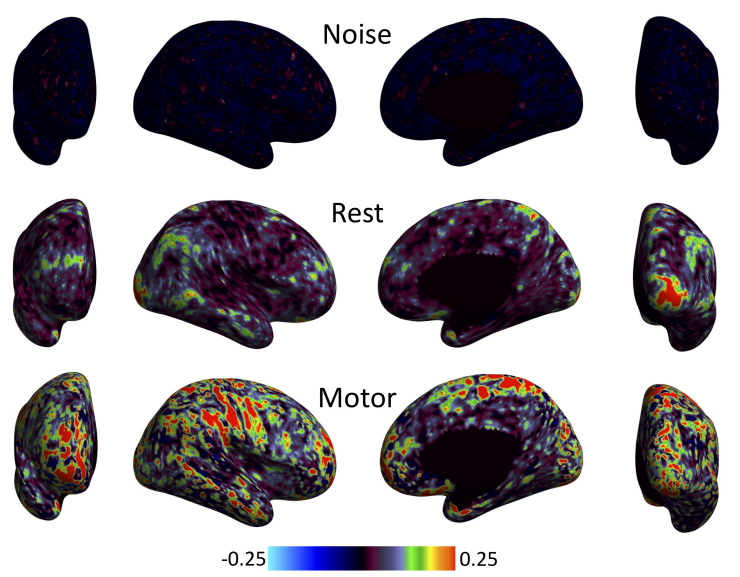

Figure 5: These are the final mitigated results using the direct low resolution approach on uniform 10k meshes and cubic interpolation shown on the brain. Note that the "Rest" image is rotated to demonstrate a clear "activation" in the occipital pole that would otherwise be obscured. One can note the difference as compared to figure 1 
bioRxiv preprint doi: https://doi.org/10.1101/2020.11.09.373860; this version posted November 9,2020 . The copyright holder for this

preprint (which was not certified by peer review) is the author/funder, who has granted bioRxiv a license to display the preprint in perpetuity. It is made available under aCC-BY-NC-ND 4.0 International license.

\section{References}

Attene, M. (2010). A lightweight approach to repairing digitized polygon meshes. The Visual computer, 26, 1393-1406. URL: http://link.springer.com/10.1007/s00371-010-0416-3 doi 10.1007/s00371-010-0416-3

Bajada, C. J., Costa Campos, L. Q., Caspers, S., Muscat, R., Parker, G. J. M., Lambon Ralph, M. A., Cloutman, L. L., \& Trujillo-Barreto, N. J. (2020). A tutorial and tool for exploring feature similarity gradients with MRI data.

- Neuroimage, 221, 117140. URL: https://linkinghub.elsevier.com/retrieve/pii/S1053811920306261 doi 10.1016/j. neuroimage.2020.117140

Bjork, J. M., Straub, L. K., Provost, R. G., \& Neale, M. C. (2017). The ABCD study of neurodevelopment: Identifying neurocircuit targets for prevention and treatment of adolescent substance abuse. Current treatment options in psychiatry, 4, 196-209. URL: http://link.springer.com/10.1007/s40501-017-0108-y doi 10.1007/s40501-017-0108-y

Brodoehl, S., Gaser, C., Dahnke, R., Witte, O. W., \& Klingner, C. M. (2020). Surface-based analysis increases the specificity

of cortical activation patterns and connectivity results. Scientific Reports, 10, 5737. URL: http://dx.doi.org/10.1038/ s41598-020-62832-z doi 10.1038/s41598-020-62832-z

Coalson, T. S., Van Essen, D. C., \& Glasser, M. F. (2018). The impact of traditional neuroimaging methods on the spatial localization of cortical areas. Proceedings of the National Academy of Sciences of the United States of America, 115, E6356-E6365. URL: http://dx.doi.org/10.1073/pnas.1801582115 doi 10.1073/pnas.1801582115.

Esteban, O., Markiewicz, C. J., Blair, R. W., Moodie, C. A., Isik, A. I., Erramuzpe, A., Kent, J. D., Goncalves, M., DuPre, E., Snyder, M., Oya, H., Ghosh, S. S., Wright, J., Durnez, J., Poldrack, R. A., \& Gorgolewski, K. J. (2019). fMRIPrep: a robust preprocessing pipeline for functional MRI. Nature Methods, 16, 111-116. URL: http://www.nature.com/articles/ s41592-018-0235-4 doi 10.1038/s41592-018-0235-4

Glasser, M. F., Smith, S. M., Marcus, D. S., Andersson, J. L. R., Auerbach, E. J., Behrens, T. E. J., Coalson, T. S., Harms, M. P., Jenkinson, M., Moeller, S., Robinson, E. C., Sotiropoulos, S. N., Xu, J., Yacoub, E., Ugurbil, K., \& Van Essen, D. C. (2016). The human connectome project's neuroimaging approach. Nature Neuroscience, 19, 1175-1187. URL: 140 http://dx.doi.org/10.1038/nn.4361 doi $10.1038 / \mathrm{nn} .4361$

Glasser, M. F., Sotiropoulos, S. N., Wilson, J. A., Coalson, T. S., Fischl, B., Andersson, J. L., Xu, J., Jbabdi, S., Webster, M., Polimeni, J. R., Van Essen, D. C., Jenkinson, M., \& Consortium, W.-M. H. (2013). The minimal preprocessing pipelines for the human connectome project. Neuroimage, 80, 105-124. URL: http://dx.doi.org/10.1016/j.neuroimage.2013.04.127 doi $10.1016 / j$.neuroimage.2013.04.127

Van Essen, D. C., Smith, S. M., Barch, D. M., Behrens, T. E. J., Yacoub, E., Ugurbil, K., \& Consortium, W.-M. H. (2013).

n The WU-minn human connectome project: an overview. Neuroimage, 80, 62-79. URL: http://dx.doi.org/10.1016/j. neuroimage.2013.05.041 doi 10.1016/j.neuroimage.2013.05.041

Van Essen, D. C., Ugurbil, K., Auerbach, E., Barch, D., Behrens, T. E. J., Bucholz, R., Chang, A., Chen, L., Corbetta, M., Curtiss, S. W., Della Penna, S., Feinberg, D., Glasser, M. F., Harel, N., Heath, A. C., Larson-Prior, L., Marcus, D., Michalareas, G., Moeller, S., Oostenveld, R., Petersen, S. E., Prior, F., Schlaggar, B. L., Smith, S. M., Snyder, A. Z., Xu, J., Yacoub, E., \& Consortium, W.-M. H. (2012). The human connectome project: a data acquisition perspective. Neuroimage, 62, 2222-2231. URL: http://dx.doi.org/10.1016/j.neuroimage.2012.02.018 doi 10.1016/j.neuroimage.2012.02.018 